\title{
COMPLEX LENGTH AND PERSISTENCE OF LIMIT CYCLES IN A NEIGHBORHOOD OF A HYPERBOLIC POLYCYCLE
}

\author{
Yu. ILYASHENKO
}

To Jaume Llibre for his 60th birthday, with best and friendly wishes

\begin{abstract}
Complex limit cycle located in a neighborhood of a hyperbolic polycycle can not vanish under a small deformation that preserves the characteristic values of the vertexes of the polycycle. The cycles either change holomorphically under the change of the parameter, or come to the boundary of the fixed neighborhood of the polycycle. The present paper makes these statements rigorous and proves them.
\end{abstract}

2010 Mathematics Subject Classification: 37F75.

Key words: Complex limit cycles, hyperbolic polycycles, eigenvalues of singular points, complex length.

\section{Introduction}

In this paper we consider planar holomorphic foliations.

\subsection{Complex limit cycles and Diophantine singular points.}

Definition 1. A characteristic number of a singular point of a complex planar foliation is a ratio of the eigenvalues of this point. A singular point is Diophantine, provided that its characteristic number $\lambda$ is negative, and there exist $C$ and $s$ such that for any irreducible fraction $\frac{p}{q}$,

$$
\left|\lambda-\frac{p}{q}\right|>\frac{C}{q^{s}} .
$$

Any singular point with a negative characteristic number has exactly two germs of invariant holomorphic curves passing through a singular point, called local separatrixes. Invariance means that the representatives of the germs with the singular point deleted belong to the leaves of the

The author was partially supported by the grants NSF 0700973 and CNRS-RFBR 10-01-93115-NTSNILa. 
foliation. These leaves (with the singular point added) are called (global) complex separatrixes.

An analytic vector field near a Diophantine singular point may be analytically linearized. Any neighborhood where the linearizing chart is well defined is called for brevity nice.

Leaves of a planar complex foliation are Riemann surfaces. They may be non-simply connected.

Definition 2. A complex cycle of a foliation is a nontrivial free homotopy class of real loops on a leaf of the foliation. This class is called a complex limit cycle (identity cycle) provided that the corresponding fixed point of the holonomy map is (is not) isolated. A real (complex) family of complex cycles with a real (respectively, complex) parameter $t$ depends continuously on $t$ provided that there exists a family of representatives of the corresponding classes of free homotopy that depend continuously on the parameter.

\subsection{Complex polycycles.}

Definition 3. A polycycle of a real vector field is a separatrix polygone, that is an oriented closed curve $\sigma$, constituted by a finite union of ordered singular points $O_{j}$ and their time oriented mutual separatrixes $\sigma_{j}$ that connect $O_{j}$ and $O_{j+1}$; sometimes, $O_{j}$ and $O_{j+1}$ may be the same. The orientation of $\sigma$ agrees with the orientation of $\sigma_{j}$.

A complex polycycle is an analogous object for a complex foliation. In this paper we deal with complex hyperbolic polycycles defined as follows.

Definition 4. A complex hyperbolic polycycle is an oriented real closed curve, that consists of a finite union of hyperbolic singular points $O_{j}$, called vertexes of the polycycle, and the real oriented connections $\sigma_{j}$ from $O_{j}$ to $O_{j+1}$, called edges of the polycycle, that belong to mutual complex separatrixes of the points $O_{j}$ and $O_{j+1}$. Again, the orientation of $\sigma$ agrees with the orientation of $\sigma_{j}$. The germs of two subsequent edges of the polycycle belong to different local separatrixes of their mutual vertex.

Consider an analytic one-parameter family of holomorphic foliations with singularities in $\mathbb{C}^{2}$ :

$$
\mathcal{F}=\left\{\mathcal{F}_{\alpha} \mid \alpha \in V, 0 \in V \subset \mathbb{C}\right\},
$$

the parameter space $V$ is bounded. Suppose that the foliation $\mathcal{F}_{0}$ has a polycycle $\sigma$ with vertexes $O_{j}$ that are Diophantine singular points. Suppose that the base $V$ is so small that the singular points $O_{j}(\alpha)$ are 
well defined. We suppose that the deformation is special: all the singular points $O_{j}(\alpha)$ are Diophantine (consequently, their characteristic numbers do not depend on $\alpha$ ).

We suppose also that any saddle connection between two singular points $O_{i}(\alpha), O_{j}(\alpha)$ is isolated in the family $\mathcal{F}$ : if such a connection $\sigma_{i j}$ occurs for $\alpha=\alpha_{0}$, then nearby foliations have no saddle connections between $O_{i}(\alpha), O_{j}(\alpha)$ that is close to $\sigma_{i j}$ for $\alpha \neq \alpha_{0}$. This implies that saddle connections between the points $O_{i}(\alpha), O_{j}(\alpha)$ occur for at most a countable number of values $\alpha \in V$.

Suppose that the neighborhoods $\mathcal{U}_{j}$ of $O_{j}$ exist such that they contain $O_{j}(\alpha)$ and are nice for all $\alpha \in V$.

\subsection{Correspondence and regular maps.}

We will now recall a construction from $[6]$ that allows us to decompose the monodromy map of a real polycycle into an product of alternating correspondence (Dulac), and regular maps. Consider a real polycycle $\sigma$ of an analytic vector field $v$ whose vertexes are hyperbolic saddles.

For any $j$, consider cross sections $\Gamma_{j}^{+}, \Gamma_{j}^{-}$in a small neighborhood of the singular point $O_{j}$ passing through the points $P_{j}^{ \pm}$chosen close to the vertexes on the edges: $P_{j}^{+} \in \sigma_{j-1}, P_{j}^{-} \in \sigma_{j}$.

Let $\Sigma_{j}^{ \pm}$be a half-interval of $\Gamma_{j}^{ \pm}$with the vertexes $P_{j}^{ \pm}$chosen in such a way that $\Sigma_{j}^{+}$and $\Sigma_{j}^{-}$belong to the same hyperbolic sector of $O_{j}$. Let $v$ be a vector field that generates the foliation near the point $O_{j}$. Then the correspondence map $\Delta_{j}: \Sigma_{j}^{+} \rightarrow \Sigma_{j}^{-}$along the phase curves of the vector field $v$ is well defined. Suppose that $v$ is linear in coordinates $x, y$ near $O_{j}$. More precisely, $v$ is orbitally analytic equivalent to a field $\left(x, \lambda_{j} y\right), \lambda_{j}<$ 0 , and $\Gamma_{j}^{ \pm}$have the form $y=c^{+}, x=c^{-}$, where $c^{+}$and $c^{-}$are some constant values. Then the correspondence map has the form:

$$
\Delta_{j}=C_{j} x^{-\lambda_{j}}
$$

for some positive $C_{j}$.

Denote by $f_{j}: \Gamma_{j}^{-} \rightarrow \Gamma_{j+1}^{+}, j=1, \ldots, n$, the map along the phase curves of $v$ that pass near the arc $\left[P_{j}^{-}, P_{j+1}^{+}\right] \subset \sigma_{j}$; the numeration is cyclic modulo $n: \Gamma_{n+1}^{+}=\Gamma_{1}^{+}$. These maps are analytic at $P_{j}^{-}$and analytically depend on the parameter $\alpha$; they are called regular maps. When we want to stress the dependence on the parameter $\alpha$, we write $\Delta_{j, \alpha}, f_{j, \alpha}$ instead of $\Delta_{j}, f_{j}$.

For the hyperbolic polycycle $\sigma$, a monodromy map $\Delta_{\sigma}: \Sigma_{1}^{+} \rightarrow \Gamma_{1}^{+}$ along the orbits of $v$ is well defined. By definition:

$$
\Delta_{\sigma}=f_{n} \circ \Delta_{n} \circ \cdots \circ f_{1} \circ \Delta_{1} .
$$


Let us now give similar definitions in the complex context. The complex correspondence maps are non-univalent, and we use a complex cycle $\gamma$ close to a complex polycycle $\sigma$ in order to choose a branch of the correspondence map.

Let $\sigma$ be a complex hyperbolic polycycle of a foliation $\mathcal{F}$, see Definition 4. On any edge $\sigma_{j}$ that connects $O_{j}$ and $O_{j+1}$, take two points $P_{j}^{-}$ and $P_{j+1}^{+}$close to $O_{j}$ and $O_{j+1}$ respectively. Take analytic cross sections $\Gamma_{j}^{ \pm}$through $P_{j}^{ \pm}$. Consider a complex cycle $\gamma$ close to $\sigma$. We say that $\gamma$ makes one circuit along $\sigma$ if $\gamma$ crosses any section $\Gamma_{j}^{ \pm}$exactly at one point $Q_{j}^{ \pm}: Q_{j}^{ \pm}=\gamma \cap \Gamma_{j}^{ \pm}$. The points $Q_{1}^{+} \in \Gamma_{1}^{+}, \ldots, Q_{n}^{+} \in \Gamma_{n}^{+}$ on the cycle are called marked, and the cycles with the marked points distinguished is called marked also. The cycle $\gamma$ is therefore split into the union of arcs

$$
\gamma_{j}=\left[Q_{j}^{+}, Q_{j}^{-}\right] \subset \gamma
$$

and

$$
\rho_{j}=\left[Q_{j}^{-}, Q_{j+1}^{+}\right] \subset \gamma .
$$

Denote by $\left(\Delta_{j}, Q_{j}^{+}\right)$a germ of a map $\left(\Gamma_{j}^{+}, Q_{j}^{+}\right) \rightarrow\left(\Gamma_{j}^{-}, Q_{j}^{-}\right)$along the leaves of $\mathcal{F}$. The analytic extension of $\left(\Delta_{j}, Q_{j}^{+}\right)$is called the (complex) correspondence or Dulac map of $\mathcal{F}$ at a point $O_{j}$. In general, it has a logarithmic branch point $P_{j}^{+}$. Denote by $\left(f_{j}, P_{j}^{-}\right)$the germ of a map $\left(\Gamma_{j}, P_{j}^{-}\right) \rightarrow\left(\Gamma_{j+1}^{+}, P_{j+1}^{+}\right)$along the leaves of $\mathcal{F}$, close to the arc $r_{j}=\left[P_{j}^{-}, P_{j+1}^{+}\right] \subset \sigma_{j}$. This is a holomorphic map at $P_{j}^{-}$; denote its representative also by $f_{j}$. This is a germ of a regular map at $P_{j}^{-}$.

When the foliations $\mathcal{F}_{\alpha}$ analytically depend on $\alpha$, the maps defined above are also analytic in $\alpha$. In this case, we add a subscript $\alpha$ to their notation.

As before, the complex cycle $\gamma$ is split into the union of the alternating $\operatorname{arcs} \gamma_{j}$ and $\rho_{j}$. Moreover:

$$
Q_{j}^{-}=\Delta_{j}\left(Q_{j}^{+}\right), \quad Q_{j+1}=f_{j}\left(Q_{j}^{-}\right) .
$$

The latter equality holds when the cycle $\gamma$ is sufficiently close to the polycycle $\sigma$. Then $Q_{1}^{+}$satisfies the following relation:

$$
Q_{1}^{+}=f_{n} \circ \Delta_{n} \circ \cdots \circ f_{1} \circ \Delta_{1}\left(Q_{1}^{+}\right) .
$$




\subsection{Dashed neighborhoods of hyperbolic complex polycycles.}

Let the family of foliations (1), the polycycle $\sigma$ and the cross sections $\Gamma_{j}$ be the same as above. Suppose that the disks $U_{j}^{ \pm} \subset \Gamma_{j}^{ \pm} \cap \mathcal{U}_{j}$ are such that for all $\alpha \in V$ the following holds:

- all the branches of the Dulac maps $\Delta_{j, \alpha}: U_{j}^{+} \rightarrow \Gamma_{j}^{-}$are well defined (below we prove that such disks exist);

- the regular maps $f_{j, \alpha}: U_{j}^{-} \rightarrow U_{j+1}^{+}$are well defined;

- the separatrixes of $\mathcal{F}_{\alpha}$ passing through $O_{j}(\alpha)$ cross the disks $U_{j}^{ \pm}$.

We call the union of the disks $U_{j}^{ \pm}$the dashed neighborhood of the polycycle $\sigma$. These neighborhoods will be used in the definition of the continuation of a complex limit cycle up to the boundary of a neighborhood of a polycycle.

\subsection{Continuation of complex limit cycles.}

For a family $\xi:[0,1] \rightarrow V, t \mapsto \alpha=\xi(t)$ we denote the image of the map $\xi$ either as $\xi(t)$ or as $\alpha(t)$.

Definition 5. Let $\gamma_{0}$ be a complex limit cycle of the foliation $\mathcal{F}_{0}$, and $\xi:[0,1] \rightarrow V, \xi(0)=0$, be a curve in the family (1). We say that the cycle $\gamma_{0}$ is extended along this curve over an arc $\left[0, t_{0}\right)$ or $\left[0, t_{0}\right]$ if there exists a continuous family $\gamma_{t}$ of complex limit cycles of the foliations $\mathcal{F}(t)=\mathcal{F}_{\alpha(t)}$ well defined for all $t \in\left[0, t_{0}\right)$ or $t \in\left[0, t_{0}\right]$ respectively. The cycle is extended over the whole curve $\xi$ if it can be extended along this curve over the arc $[0,1]$.

Definition 6. The family of complex limit cycles in the previous definition is marked provided that representatives of the cycles $\gamma_{t}$ (still denoted by $\gamma_{t}$ ) exist such that they intersect all the $\operatorname{discs} U_{j}^{ \pm}$exactly once at the points $Q_{j}^{ \pm}(t)$. The family above is continued up to the boundary of the dashed neighborhood $\cup U_{j}^{ \pm}$of the polycycle $\sigma$ provided that all the points $Q_{j}^{ \pm}(t), t<t_{0}$, belong to the interior of the disc $U_{j}^{ \pm}$, and at least one of the points $Q_{j}^{ \pm}\left(t_{0}\right)$ belongs to the boundary of the corresponding disc.

Definition 7. A complex cycle $\gamma_{0}$ has a generalized $\varepsilon$-lift property in a dashed neighborhood of the polycycle, provided that the following holds. For any curve $\xi:[0,1] \rightarrow V, \xi(0)=0$, and any $\varepsilon>0$, there exists a curve $\xi_{\varepsilon}, \varepsilon$-close to $\xi$ in $C_{[0,1]}, \xi_{\varepsilon}(0)=0$, and such that the marked cycle $\gamma_{0}$ persists under the continuation along $\xi_{\varepsilon}$ in the following sense. The cycle $\gamma_{0}$ may be either extended over the whole curve $\xi_{\varepsilon}$, or along this curve over an arc $\left[0, t_{0}\right]$ up to the boundary of the dashed neighborhood. 


\subsection{Results.}

Theorem 1. Consider a family (1) of foliations described above. Let the polycycle $\sigma$, the complex limit cycle $\gamma$ and the dashed neighborhood be the same as above. Then the cycle $\gamma$ has the generalized $\varepsilon$-lift property in the family (1).

The proof is based on the notion of the complex length of a complex limit cycle that we now introduce. Denote by $\left(\zeta_{j, \alpha}, \eta_{j, \alpha}\right)$ the linearizing chart for $\mathcal{F}_{\alpha}$ near $O_{j}(\alpha)$. Let $\zeta_{j, \alpha}$ vanish on the separatrix of $O_{j}(\alpha)$ that intersects $U_{j}^{+}$.

Definition 8. Consider a family of the marked complex limit cycles $\gamma_{t}$ that cross the discs $U_{j}^{+}$of the dashed neighborhood at the marked points $Q_{j}^{+}(t)$. Let $\eta$ be a segment $\left[0, t_{0}\right]$. Complex length of the cycle $\gamma_{t}$ in the family $\left\{\gamma_{t} \mid t \in \eta\right\}$ is a holomorphic vector function

$$
\begin{gathered}
L:=\left(L_{1}, \ldots, L_{n}\right), \\
L_{j}(t)=\log \zeta_{j, \alpha(t)}\left(Q_{j}^{+}(t)\right), \\
\arg \zeta_{j, \alpha(0)}\left(Q_{j}^{+}(0)\right) \in[-\pi, \pi) .
\end{gathered}
$$

Definition 9. For the same family, the modified complex length of the cycle $\gamma_{t}$ in the family $\left\{\gamma_{t} \mid t \in \eta\right\}$ is

$$
l(t):=\sum_{1}^{n}\left|L_{j}(t)\right|
$$

The Euclidean length of the cycle $\gamma_{t}$ from Definition 8 is the minimal length of its representative that contains the marked point $Q_{j}^{+}(t)$.

Remark 1. The Euclidean length of the cycle does not depend on the family in which the cycle is included. On the contrary, the complex length makes sense only when the cycle is included in a family. The extension of $L(t)$ over a segment $\eta$ allows us to choose the proper branch of the logarithm in (7).

Remark 2. The complex length depends on the choice of coordinates $\zeta_{\alpha}$. Yet an analytic change of coordinates: $\omega_{\alpha}=F\left(\zeta_{\alpha}, \alpha\right), \omega_{\alpha}\left(O_{\alpha}\right)=0$, results in a bounded change of the vector function $L$ on all of its domain for any curve $\xi:[0,1] \rightarrow V$. The same is true for the modified length.

Theorem 2. The modified complex length of the limit cycle $\gamma_{t}$ majorizes its Euclidean length $\left|\gamma_{t}\right|$. This means that there exists a positive constant $C>0$ depending on the family $\left\{\gamma_{t}\right\}$ such that

$$
\left|\gamma_{t}\right|<C(l(t)+1) \text {. }
$$


The modified complex length may take zero values. For this reason, the free term in the right-hand side of (9) is inserted.

Theorem 2 implies Theorem 1. Theorem 2 is proved in Section 2 and Theorem 1 in Section 3.

Before proving the results stated above, let us make a brief survey.

\subsection{Persistence theorems for complex dynamical systems.}

Theorem 1 is a part of a vast realm of persistence problems for parameter depending complex dynamical systems.

Problem 1. Is it correct that complex limit cycles in the family of polynomial equations

$$
\dot{z}=P_{n}(z), \quad z \in \mathbb{C}^{2}
$$

have an $\varepsilon$-lift property?

This means that for any curve $\xi:[0,1] \rightarrow \mathcal{A}_{n}$, where $\mathcal{A}_{n}$ is the space of coefficients of equations (10), for any complex limit cycle $\gamma$ of equation $\xi(0)$ and any $\varepsilon>0$, there exists a curve $\xi_{\varepsilon}, \xi_{\varepsilon}(0)=\xi(0)$, $\varepsilon$-close to $\xi$ in $C_{[0,1]}$, such that a continuous family $\gamma_{t}$ is well defined for $t \in[0,1]$, where $\gamma_{0}=\gamma$, and $\gamma_{t}$ a complex limit cycle of the equation $\xi_{\varepsilon}(t)$.

Consider a real limit cycle of a real planar polynomial vector field. It is a complex limit cycle for the complexification of the corresponding differential equation. We say that this complex cycle is generated by a real one. Problem 1, for complex limit cycles generated by real ones, was stated in $[7]$ in slightly different terms. The problem stays unsolved. Theorem 1 above is a solution for a particular case of this problem.

A global persistence result for another class of dynamical systems is obtained in $[\mathbf{1}]$. It claims that heteroclinic points of periodic orbits of polynomial automorphisms of $\mathbb{C}^{2}$ may be globally extended over the parameter space. Note that periodic orbits of polynomial automorphisms depend algebraically on the coefficients. Therefore, they are globally defined as functions of parameters, and in particular, have the $\varepsilon$-lift property. But their heteroclinic points are transcendental functions of the parameters, and $\varepsilon$-lift property for them requires new tools to be developed. These tools were elaborated in [1], modified in [4], and used in this paper.

Some persistence problems are stated in [5]. One of them asks whether the complex Poincaré map is globally extendable for generic planar complex polycycle foliations. Some degenerate foliations may have a Poincaré map non-extendable beyond some disc [2]. This shows, how challenging 
Problem 1 is. At present, it is difficult to predict whether or not the answer to Problem 1 is positive.

After this brief survey, let us turn to the proof of our main results.

\section{Complex length vs Euclidean length}

In this section we prove Theorem 2.

\subsection{A relation between correspondence and monodromy maps.}

Recall that a monodromy map of a separatrix of a complex singular point is a holonomy map that corresponds to a loop on the separatrix that makes one circuit around the singular point in the positive direction.

Let $\Delta_{j, \alpha}: \Gamma_{j}^{+} \rightarrow \Gamma_{j}^{-}$be the correspondence (Dulac) map along the leaves of $\mathcal{F}_{\alpha}$. It is not a univalent map; fix some branch $\Delta_{j, \alpha}^{0}$ of it on $\Gamma_{j}^{+}$ cut along the negative ray in some coordinate $x$. Denote by $e^{2 \pi i k} x$, $x \in \mathbb{C}$, the endpoint of an arc on the universal cover over $\mathbb{C}^{*}$ that covers the curve in $\mathbb{C}^{*}$ emanating from $x$, making $k$ turns around 0 and coming back to $x$. Let $M_{j}(\alpha): \Gamma_{j}^{-} \rightarrow \Gamma_{j}^{-}$be the monodromy map of the local separatrix of $O_{j}(\alpha)$ that crosses $\Gamma_{j}^{-}$at the point $P_{j}^{-}$. In these notations, for any $k \in \mathbb{Z}$,

$$
\Delta_{j, \alpha}\left(e^{2 \pi i k} x\right)=M_{j}^{k}(\alpha) \circ \Delta_{j, \alpha}^{0}(x) .
$$

In the linearizing coordinates this is trivial; but the relation holds even if these coordinates do not exist. Namely, it holds true for any non-degenerate singular point of a complex foliation, whose characteristic number $\lambda$ lies in the left halfplane: $\Re \lambda<0,[\mathbf{3}]$.

Let us state analytic counterpart of this formula. Let, as before, $\zeta_{j, \alpha}, \eta_{j, \alpha}$ be a linearizing chart near $O_{j}(\alpha)$. Let $x_{j, \alpha}=\left.\zeta_{j, \alpha}\right|_{\Gamma_{j}^{+}}, y_{j, \alpha}=$ $\left.\eta_{j, \alpha}\right|_{\Gamma_{j}^{-}}$. Let $\lambda_{j}$ be the characteristic number of $O_{j}$ (the eigenvalue corresponding to the local separatrix that contains $\left(\sigma_{j}, 0\right)$ is in the denominator), and $\nu_{j}=e^{2 \pi i \lambda_{j}}$. The separatrix of $O_{j}(\alpha)$ that contains the germ $\left(\sigma_{j-1}(\alpha), O_{j}(\alpha)\right)$ or $\left(\sigma_{j}(\alpha), O_{j}(\alpha)\right)$ is called incoming (respectively, outcoming) separatrix.

For a linear vector field with a real characteristic number, the monodromy map $M_{j}$ of the outcoming separatrix $W_{j}^{-}$of $O_{j}$ is well defined:

$$
\Gamma_{j}^{+} \rightarrow \Gamma_{j}^{-}, \quad y_{j, \alpha} \mapsto \nu_{j} y_{j, \alpha} .
$$

As $\lambda_{j}$ is real, $\left|\nu_{j}\right|=1$, and in the coordinate $y_{j, \alpha}, M_{j}$ is a rigid rotation. Thus all the iterates of $M_{j, \alpha}$, positive or negative, are well defined in $\Gamma_{j}^{-}$. Note that $M_{j, \alpha}$ does not dependent on $\alpha$ in the normalizing coordinates, but it may well depend on $\alpha$ in the original coordinates. 
The correspondence map in the normalizing coordinates has the form:

$$
x_{j, \alpha} \mapsto y_{j, \alpha}=x_{j, \alpha}^{-\lambda_{j}} .
$$

In this coordinates relation (11) is a simple property of branching of a power function; but it gives an important geometric interpretation of this property that will be used below.

\subsection{Majorizing the length.}

As in Definition 8, consider a real family of the marked complex limit cycles $\gamma_{t}$ that cross the discs $U_{j}^{+}$of the dashed neighborhood at the marked points $Q_{j}^{+}(t)$. Denote by $v_{j}(t)$ the argument of $x_{j}(t):=$ $\zeta_{j, \alpha(t)}\left(Q_{j}^{+}(t)\right)$ extended from $t=0$ continuously in $t$; we suppose that $v_{j}(0) \in[-\pi, \pi]$. Let $|\gamma|$ be the length of the curve $\gamma$. Denote by $\gamma_{j, t}$ the arc of $\gamma_{t}$ that connects $Q_{j}^{+}(t)$ and $Q_{j}^{-}(t)$. We will prove below the following relation:

$$
\left|\gamma_{j, t}\right|<C_{0}+C_{1}\left|v_{j}(t)\right| .
$$

So, the Eucledian lengths of the $\operatorname{arcs} \gamma_{j, t}$ are majorized by the arguments $v_{j}$. Hence, the sum of lengths of the arcs $\gamma_{j, t}$ is majorized by the complex length of $\gamma_{t}$; the lengths of $\rho_{j, t}$ stay bounded. This implies Theorem 2, modulo relation (14).

\subsection{Proof of relation (14).}

Relation (14) follows from (11). Consider first a neighborhood of a hyperbolic singular point $O$ of a foliation $\mathcal{F}$ where the linearizing chart $(z, w)$ is well defined, and consider a cross-section $\Gamma^{-}$to a separatrix with the chart $y=\left.w\right|_{\Gamma^{-}}$. In a neighborhood $\left(\Gamma^{-}, 0\right)$ the monodromy map $M$ is well defined. In a normalizing chart it is a rigid rotation, and all its iterates are well defined in one and the same domain.

For any $y \in\left(\Gamma^{-}, 0\right)$ let $M(y)$ be its image under the monodromy map, and $\lambda(y)$ be an arc on the leaf of the foliation $\mathcal{F}$ that connects $y$ and $M(y)$, and is projected along the $w$ axis to a positively oriented circle $S$ centered at zero on the $z$ axis. There exists $C$ such that $|\lambda(y)|<$ $C$ uniformly in $\left(\Gamma^{-}, 0\right)$.

Below we use the universal constant $C$; one and the same symbol $C$ corresponds to different values.

Consider a cross-section $\Gamma^{+}$to the other complex separatrix, and let $\Delta: \Gamma^{+} \rightarrow \Gamma^{-}$be the correspondence map of $\mathcal{F}$. Let $\Gamma_{\text {cut }}^{+}$be a disc on the cross-section $\Gamma^{+}$cut along the negative ray with two edges of the cut included. This is a compact set; suppose that it is so small that the 
correspondence map $\Delta: \Gamma_{\text {cut }}^{+} \rightarrow \Gamma^{-}$is well defined. For any $x \in \Gamma_{\text {cut }}^{+}$ let $\delta(x)$ be an arc on the leaf of $\mathcal{F}$ that connects $x$ and $\Delta(x)$. Then there exists $C$ such that $|\delta(x)|<C$ uniformly in $x \in \Gamma_{\text {cut }}^{+}$. The branch of the correspondence map thus defined is denoted by $\Delta^{0}$.

Let us now pass to relation (14). Consider a curve $\xi:[0,1] \rightarrow V$ and suppose that the cycle $\gamma_{0}$ may be extended along the whole curve $\xi$ up to a family of cycles $\gamma_{t}$ with the marked points $Q_{j}^{+}(t)$. Let us estimate the length of the curve $\gamma_{j, t}$, see Subsection 2.2. We will do that for $j=1$. Case of arbitrary $j$ is treated in the same way. Let $x_{1}(t)$ be the same as in the previous subsection. Consider the curve $\zeta_{1}: t \mapsto x_{1}(t)$. The curve $\zeta_{1}$ begins at $x_{1}(0)$ and ends at $x_{1}(1)$. Let us connect the points $x_{1}(1)$ and $x_{1}(0)$ by a curve $\zeta_{0} \subset \Gamma_{\text {cut }}^{+}$. The curve $\eta=\zeta_{1} \zeta_{0}$, still parametrized by $[0,1]$, makes $k$ turns around zero; the number $k$ is defined by the formula:

$$
|\arg \eta(1)-\arg \eta(0)-2 \pi k| \leq \pi .
$$

By (11), the curve $\gamma_{1,1}=\gamma_{1, t}$ for $t=1$, may be chosen as an arc $\left(x(1), \Delta_{1}^{0}(x(1))\right)$, continued by an arc $\lambda_{1, k}$ which is defined as the $k$-fold cover on the leaf over the circle $S$ mentioned in the first paragraph of this subsection. Hence, for some $C$,

$$
\left|\gamma_{1,1}\right| \leq C(k+1) \leq C\left(\frac{\left|\arg x_{1}(1)\right|}{2 \pi}+1\right) .
$$

This proves (14) for $j=1$. The proof for arbitrary $j$ is the same, as mentioned above.

Thus, the proof of Theorem 2 is completed.

\section{Persistence of limit cycles}

Here we deduce Theorem 1 from Theorem 2, and from the boundary properties of analytic functions.

\subsection{Persistence domains for limit cycles.}

Let us first describe the domain to which the complex length of the limit cycle may be analytically extended.

Recall that a complex cycle $\gamma$ in a family of foliations $V$, see (1) is called marked if it is represented by a loop that crosses exactly once every disc of the dashed neighborhood of the polycycle $\sigma$.

We now modify the definition of the persistence domain for a complex limit cycle from [4], in order to adjust it to the local cross-sections $U_{j}^{+} \subset$ $\Gamma_{j}^{+}$. In what follows we make use of the marked points $Q_{j}^{+}$only. 
Definition 10. The persistence domain for complex limit cycle $\gamma$ in the family (1) is a set that consists of marked complex cycles (limit or with holonomy identity) of the foliations $\mathcal{F}_{\alpha}, \alpha \in V$, see (1), with the marked points $Q_{j}^{+} \in U_{j}^{+}$, and has the following properties:

- path connectedness: any representative of a cycle that belongs to the persistence domain may be connected to $\gamma_{0}$ by a homotopy whose elements are representatives of marked limit cycles of foliations $\mathcal{F}_{\alpha}$ with marked points located in $U_{j}^{+}$;

- maximality: the persistence domain is not contained in a larger set with the above property.

Let $U=U_{1}^{+} \times \cdots \times U_{n}^{+}$. The persistence domain defined above is denoted by $L C=L C(\mathcal{F}, U, \gamma)$. Its natural projection $p$ onto the Cartesian product $\mathcal{W}=V \times U$ is well defined: each marked cycle $\gamma^{\prime}$ from the persistence domain is projected to the parameter $\alpha$ of the corresponding foliation, and to the tuple of the marked points of $Q(\alpha)=$ $\left(Q_{1}^{+}(\alpha), \ldots, Q_{n}^{+}(\alpha)\right)$ in $U$. The image of the projection $p$ is locally an analytic set, and the projection is locally one-to-one. Indeed, consider a parameter depending tuple of complex Poincaré maps of $\gamma^{\prime}$ corresponding to the tuple of the cross sections $U_{j}^{+}$. This tuple of maps may be considered as one parameter depending vector Poincaré map $U \rightarrow \Gamma_{1}^{+} \times \cdots \times \Gamma_{n}^{+}$. The points $\left(\alpha, Q_{1}^{+}(\alpha), \ldots, Q_{n}^{+}(\alpha)\right) \in \mathcal{W}$ are the fixed points of this map. Hence, they form an analytic set. Therefore, $Z=p(L C)$ is a dimension one complex variety immersed in $V \times U$. Note that in general $Z$ is not an analytic subset of $V \times U$, because it may not be closed.

Projection $p$ provides an analytic structure to the persistence domain of a limit cycle: the local analytic structure on $Z$ is pulled back onto $L C$. Note that $p$ is locally a bijection, but may be not one to one globally: the projection $p$ may have a nontrivial holonomy. Let $L C_{\mathcal{W}}$ be an irreducible component of the set $p^{-1}(\mathcal{W})$ that contains $\gamma$, and $\partial L C_{\mathcal{W}}=p^{-1}(V \times$ $\partial U) \cap C l L C_{\mathcal{W}}$.

Consider a projection $\pi_{U}: L C_{\mathcal{W}} \rightarrow U$ that brings any cycle from $L C_{\mathcal{W}}$ to the tuple of its marked points in $U$. Consider a natural map $\pi_{V}: L C_{\mathcal{W}} \rightarrow V$. Note that $p=\pi_{V} \times \pi_{U}$.

Theorem 1 is equivalent to a statement that projection $\pi_{V}$ has a modified $\varepsilon$-lift property in sense of the following definition.

Definition 11. A projection $\pi$ of an analytic set $X$ with a boundary $\partial X$ on an analytic set $Y \subset \mathbb{C}^{n}$, with metric on $Y$ induced from $\mathbb{C}^{n}$, has a modified $\varepsilon$-lift property provided that the following holds. For any $\varepsilon>0$, 
$p \in Y, q \in X, \pi(q)=p$, and any curve $\xi:[0,1] \mapsto Y, \xi(0)=p$, there exists a curve $\xi_{\varepsilon}, \varepsilon$-close to $\xi$ in the $C_{[0,1]}$ metric such that:

- either $\xi_{\varepsilon}$ may be lifted to $X$ starting at $q$, that is there exists a curve $\widehat{\xi}_{\varepsilon}:[0,1] \rightarrow X, \widehat{\xi}(0)=q, \pi \widehat{\xi}_{\varepsilon}=\xi_{\varepsilon}$;

- or there exists $t_{0}$ such that the curve $\left.\xi_{\varepsilon}\right|_{\left[0, t_{0}\right]}$ may be lifted to $X \cup \partial X$ as a curve $\widehat{\xi}_{\varepsilon}$ starting at $q$, and $\widehat{\xi}_{\varepsilon}\left(t_{0}\right) \in \partial X$.

This definition reproduces a parallel definition from [1] with a modification due to the existence of the boundary $\partial X$.

In what follows, we will prove that the projection $\pi_{V}: L C_{\mathcal{W}} \rightarrow V$ has a modified $\varepsilon$-lift property.

\subsection{Bounded length and extension of limit cycles.}

Lemma 1. Consider a real analytic one-parameter subfamily of foliations $\mathcal{F}_{\alpha(t)}, t \in[0,1]$, in the family (1), and a family of limit cycles $\gamma_{t}$ of these foliations defined on a semi-interval $t \in\left[0, t_{0}\right)$ in the parameter space. Suppose that the length $\left|\gamma_{t}\right|$ does not tend to infinity as $t \rightarrow t_{0}$. Then either the family $\gamma_{t}$ tends to a complex polycycle $\gamma$ as $t \rightarrow t_{0}$ or the family $\gamma_{t}$ may be extended to $t_{0}$ and beyond.

Proof: By assumption, there exists a sequence $t_{i} \rightarrow t_{0}$ such that the lengths $\left|\gamma_{t_{i}}\right|$ are bounded. A sequence of piecewise smooth curves of bounded length contains a convergent subsequence: $\gamma_{t_{i}} \rightarrow \gamma^{0}$ (in the Hausdorff sense) as $t_{i} \rightarrow t_{0}$, where $\gamma^{0}$ is again a closed curve of a finite length.

Suppose first that the curve $\gamma^{0}$ contains no singular point of the foliation $\mathcal{F}_{\alpha\left(t_{0}\right)}$. All the curves $\gamma_{t_{i}}$ belong to some leaves of the foliations $\mathcal{F}_{\alpha\left(t_{i}\right)}$. By the continuous dependence of the leaves on the parameter, $\gamma^{0}$ belongs to some leaf of $\mathcal{F}_{\alpha\left(t_{0}\right)}$ because it contains no singular points. Consider the Poincaré map of the cycle $\gamma^{0}$ corresponding to $U_{1}^{+}$. It is a germ of a holomorphic map near its fixed point in $V \times U$. The set of the fixed points of this germ contains the points corresponding to $\gamma_{t_{i}}:\left(\alpha\left(t_{i}\right), Q_{1}^{+}\left(t_{i}\right)\right)$. The persistence domain of the cycle $\gamma^{0}$ has therefore a nonempty intersection with that of the cycle $\gamma_{0}$. Adding the first domain to the second one will preserve the properties listed in Subsection 3.1. By maximality, the first domain belongs to the second one. Hence, the family of limit cycles $\gamma_{t}, t \in\left[0, t_{0}\right)$ is extended to $t_{0}$ and beyond. This proves Lemma 1 in the case when $\gamma^{0}$ contains no singular points of the foliation $\mathcal{F}_{\alpha\left(t_{0}\right)}$.

Suppose now that the curves $\gamma^{0}$ contains some singular points of $\mathcal{F}_{\alpha\left(t_{0}\right)}$. Then they are some of the points $O_{j}\left(\alpha_{0}\right)$ that evolve from the vertexes 
of the polycycle $\gamma$. Any arc of $\gamma^{0}$ between two singular points belongs to a leaf of $\mathcal{F}_{\alpha\left(t_{0}\right)}$. Hence, $\gamma^{0}$ is a polycycle. This completes the proof of Lemma 1.

Note that there is but a countable number of values of $\alpha \in V$ for which $\mathcal{F}_{\alpha}$ has a polycycle, as mentioned in Subsection 1.2.

Remark 3. The idea to use bounded length of complex limit cycles for the extention above goes back to [7].

\subsection{Projection of the persistence domain and its universal cover.}

Consider a universal cover $\widehat{L C}_{\mathcal{W}}$ over $L C_{\mathcal{W}}$ with the base point $\gamma$ and with the projection $\widehat{\pi}: \widehat{L C}_{\mathcal{W}} \rightarrow L C_{\mathcal{W}}$. The complex length (still denoted by $L$ ) is well defined on $\widehat{L C}_{\mathcal{W}}$. The definition is the following. Any point $\widehat{\gamma} \in \widehat{L C}_{\mathcal{W}}$ corresponds to a homotopy class of families of limit cycles in $L C_{\mathcal{W}}$; the families of this class have the same initial point that coincides with the base point $\gamma$ of the cover, and the same endpoint $\widehat{\pi}(\widehat{\gamma})$.

Take a representative of this class, namely, a family $\gamma_{t}$ of limit cycles, $t \in[0,1], \gamma_{0}=\gamma$. By definition, $\gamma_{t}$ is a complex limit cycle of a foliation $\mathcal{F}_{\alpha(t)}$, where $\alpha(t)=\pi_{V} \gamma_{t}$. For such a family, the complex length of $\gamma(1)$, see $(7)$, is well defined. Now, note that $\widehat{\pi}(\widehat{\gamma})=\gamma(1)$, and let the complex length at $\widehat{\gamma}$ be equal to

$$
L(\widehat{\gamma})=L(\gamma(1))
$$

This definition does not depend on the representative, because under the homotopy, the value $\zeta_{j, \alpha(t)}\left(Q_{j}^{+}(t)\right)$ in $(7)$ is nonzero.

Remark 4. The persistence domain of a limit cycle $\gamma_{0}$ may be not simply connected. Therefore, two paths that connect a cycle $\gamma$ with the original cycle $\gamma_{0}$ may produce two different values of the complex length of $\gamma$. Yet on the universal cover $\widehat{L C}_{\mathcal{W}}$ the complex length is well defined.

Let

$X=\left\{\pi_{X}(\widehat{\gamma}):=\left(\pi_{U} \circ \widehat{\pi}(\widehat{\gamma}), \pi_{V} \circ \widehat{\pi}(\widehat{\gamma}), L(\widehat{\gamma})\right) \mid \widehat{\gamma} \in \widehat{L C}_{\mathcal{W}}\right\}, \quad X \subset \mathbb{C}^{2 n+1}$.

Proposition 1. The set $X$ is a closed analytic subset of $U \times V \times\left(\mathbb{C}^{-}\right)^{n} \subset$ $\mathbb{C}^{2 n+1}, \mathbb{C}^{-}=\{\lambda \mid \Re \lambda<0\}$.

Proof: Take a sequence $x_{k} \in X$ that converges to a point $x^{0} \in U \times V \times$ $\left(\mathbb{C}^{-}\right)^{n}$. Let $x_{k}=\left(Q_{k 1}^{+}, \ldots, Q_{k n}^{+}, \alpha_{k}, L^{k}\right)=\pi_{X}\left(\widehat{\gamma}_{k}\right)$, and $\widehat{\pi} \widehat{\gamma}_{k}=\gamma_{k}$. By definition, $\gamma_{k}$ is a complex limit cycle of the foliation $F_{\alpha_{k}}$ with the marked points $Q_{k 1}^{+}, \ldots, Q_{k n}^{+}$. Its complex length in the family that corresponds to $\widehat{\gamma}_{k}$ is a complex vector $L^{k}$. 
The convergence $x_{k} \rightarrow x^{0}$ implies that the sequence $L^{k}$ is bounded. By Theorem 2, the sequence of the Euclidean lengths of the cycles $\gamma_{k}$ is bounded. Hence, by Lemma 1, the sequence $\gamma_{k}$ has a subsequence $\gamma_{k_{l}}$ that converges either to a complex limit cycle or to a polycycle $\gamma^{0}$. Below we show that the second case is impossible. Consider the first one.

The same arguments as at the end of the proof of Lemma 1 imply that $\gamma^{0} \in L C_{\mathcal{W}}$. Then $\widehat{\gamma}_{k_{l}} \rightarrow \widehat{\gamma}^{0} \in \widehat{L C}_{\mathcal{W}}$, and $x^{0}:=\pi_{X}\left(\widehat{\gamma}^{0}\right) \in X$.

Suppose now that $\gamma^{0}$ is a polycycle. Then the subsequence $\gamma_{k}$ approaches some singular point. The marked points of these cycles that belong to the incoming separatrix tend to zero in the chart used for the definition (7) of the complex length. Therefore, the complex lengths $L^{k}$ of the cycles $\gamma_{k}$ tend to infinity, that contradicts the assumption that the sequence $L^{k}$ is bounded.

\subsection{Tameness on disks and $\varepsilon$-lifts.}

The following property is sufficient for the modified $\varepsilon$-lift property to hold.

Definition 12. Let $X$ be an analytic subset with boundary of the product of two spaces $\mathbb{C}^{n} \times \mathbb{C}^{m}$ with the natural projection $\pi$ of the product along the second factor onto the first one. Let $Y=\pi X$. The set $X$ is tame in disks over $Y$ provided that for any holomorphic map $\Phi: D \rightarrow X$ of an open unit disk such that $\pi \circ \Phi(D)$ is bounded, the radial limit

$$
\lim _{r \rightarrow 1} \Phi\left(r e^{i \theta}\right)=x(\theta)
$$

exists for a.e. $\theta$. We also say that the map $\pi: X \rightarrow Y$ is tame on disks.

Lemma 2. A map $\pi: X \rightarrow Y$ of a one-dimensional analytic set with a boundary to a one-dimensional analytic set $Y$, has a modified $\varepsilon$-lift property, provided that $\pi$ is tame on disks.

Proof: The proof is similar to the proof of Lemma 1 from [4]. We reproduce it here because the presence of the boundary requires some modifications. Suppose that the lemma is wrong. Then by Definition 11, there exist two points $p \in Y, q \in X$, a curve $\xi:[0,1] \rightarrow Y, \xi(0)=p=\pi(q)$, and $\varepsilon>0$, see Definition 11, with the following property. For every curve $\xi_{\varepsilon}:[0,1] \rightarrow Y, \xi_{\varepsilon}(0)=p$ which is $\varepsilon$-close to $\xi$, neither there exists a lift $\widehat{\xi}_{\varepsilon}$ of $\xi_{\varepsilon}$ to $X: \widehat{\xi}_{\varepsilon}(0)=q, \pi \widehat{\xi}_{\varepsilon}=\xi_{\varepsilon}$, nor there exists $t_{0}$ such that the curve $\left.\xi_{\varepsilon}\right|_{\left[0, t_{0}\right]}$ may be lifted to $X \cup \partial X$, and $\widehat{\xi}_{\varepsilon}\left(t_{0}\right) \in \partial X$. We will bring this assumption to a contradiction with the hypothesis that $X$ is tame in disks over $Y$. 
Without loss of generality we may assume that the map $\xi$ is analytic and may be extended to some neighborhood $W$ of $[0,1]$ in $\mathbb{C}$. Consider the inverse image of the projection: $\pi^{-1}(W) \subset X$. Let $S_{q}$ be its irreducible component that contains $q$. The set $S_{q}$ is again an analytic set with a boundary.

As the lemma is assumed to be wrong, there exist the following objects: a positive number $\varepsilon$, a cover of $[0,1]$ by $\varepsilon$-disks in $W$, two disks $D^{\prime} \subset W, D^{\prime \prime} \subset W$ of this cover and two points $p^{\prime} \in D^{\prime}, q^{\prime} \in S_{q}$ with the following property. The projection $\pi q^{\prime}$ equals $p^{\prime}$, and no curve $\gamma\left(p^{\prime}, p^{\prime \prime}\right) \subset D^{\prime}, p^{\prime \prime} \in \partial D^{\prime} \cap D^{\prime \prime}$ may be lifted to $S_{q}$ with the initial point $q^{\prime}$ up to the end of the curve or up to the boundary of $X$. We will refer to this statement as $D^{\prime}, D^{\prime \prime}$-property.

Let $S$ be the irreducible component of the intersection $\pi^{-1} D^{\prime} \cap S_{q}$, that contains $q^{\prime}$. Let $\widehat{S}$ be the universal cover over $S$ with the base point $q^{\prime}$ and projection $\widehat{\pi}: \widehat{S} \rightarrow S$. Let $\widehat{\Phi}: D \rightarrow \widehat{S}$ be the uniformization of $\widehat{S}$ and $\widehat{\Phi}(0)=q^{\prime}$.

We will need the following definition.

Definition 13. Let $\Omega$ be a bounded domain in $\mathbb{C}, \Psi: D \rightarrow \Omega-$ a holomorphic function, and let $\eta:[0,1] \rightarrow \bar{D}$ be a curve such that $\eta([0,1)) \subset D$ and $\eta(1) \in \partial D$. The curve $\eta$ is called an interior end for $\Psi$ and $\Omega$ provided that the limit

$$
z=\lim _{r \rightarrow 1} \Psi \circ \eta(r)
$$

exists, and $z \in \Omega$.

The following proposition provides a sufficient condition for the existence of interior ends for a map of one disc onto another.

Proposition 2. Let $D$ and $D^{\prime}$ be two copies of the unit disc, and $\Psi: D \rightarrow D^{\prime}$ be a non-constant holomorphic function. Let $\overline{\Psi(D)} \not \supset \bar{D}^{\prime}$. Then there exists a set of angles of positive measure such that the corresponding set of radii are the interior ends for $\Psi$ and $D^{\prime}$.

Proof: Suppose that the proposition fails. Then the difference $\partial D^{\prime} \backslash$ $\overline{\Psi(D)}$ contains an open arc $A$, and, at the same time, the set of angles for which the correspondent radii, oriented from the center, are the interior ends, has measure zero.

Consider a holomorphic map $\Psi^{+}: D^{\prime} \rightarrow D^{+}$, where $D^{+}$is a half-disc $D^{\prime} \cap\{\operatorname{Im} z \geq 0\}$, that brings the arc $A$ to a semi-circle in the boundary of $D^{+}$. Then $\Psi^{+}\left(\overline{\Psi(D)} \cap \partial D^{\prime}\right) \subset[-1,1]$. The function $f=\Psi^{+} \circ \Psi$ is holomorphic and bounded. Hence, by the Fatou Theorem, it has radial limits 
almost everywhere. By assumption, these limit values belong to the diameter $[-1,1]$. Hence, the bounded harmonic function $v=\operatorname{Im} f: D \rightarrow \mathbb{R}$ has a radial limit zero for a.e. radius. By the Poisson formula, $v \equiv 0$. Hence, $f \equiv$ const, $\Psi \equiv$ const, a contradiction.

Let us turn back to the proof of Lemma 2. Consider a map

$$
\Psi: D \rightarrow D^{\prime}, \quad \Psi=\pi \circ \widehat{\pi} \circ \widehat{\Phi} .
$$

Here $D^{\prime}$ is the disc from the $\left(D^{\prime}, D^{\prime \prime}\right)$-property above. It may be identified with a unit disc. By the $\left(D^{\prime}, D^{\prime \prime}\right)$-property, $\Psi$ satisfies the assumptions of Proposition 2, because $\overline{\Psi(D)} \cap D^{\prime} \cap D^{\prime \prime}=\emptyset$. By this proposition, the set of radii that are the interior ends for the map $\Psi$ and the domain $D^{\prime}$, has a positive measure.

By assumption, the set $X$ is tame in disks over $Y$. Consider a map

$$
\Phi: D \rightarrow X, \quad \Phi=\widehat{\pi} \circ \widehat{\Phi} .
$$

Hence, for almost every $\theta \in S^{1}$, the curve

$$
\lambda_{\theta}:[0,1] \rightarrow X, \quad r \rightarrow \Phi\left(r e^{i \theta}\right)
$$

has a limit $x(\theta)=\lim _{r \rightarrow 1} \lambda_{\theta}(r)$. As the set of interior ends has positive measure, we can suppose that the radius corresponding to such $\theta$ is an interior end. Take and fix one of such $\theta$. The point $x(\theta) \in X \cup$ $\partial X$, because the latter union is closed. Note that $x(\theta) \notin \partial X$ by the assumption in the first paragraph of the proof of Lemma 2. Hence, $x(\theta) \in X$, and $\pi x(\theta) \in D^{\prime}$, because the corresponding radius is an interior end for $\Phi$. Therefore, $x(\theta) \in S$.

This contradicts to the definition of the map $\widehat{\Phi}$. Indeed, consider the cover $\widehat{\lambda}_{\theta}$ over $\lambda_{\theta}$ with the base point $q^{\prime}, \widehat{\lambda}_{\theta}(r)=\widehat{\Phi}\left(r e^{i \theta}\right)$. As $\lambda_{\theta}(r) \rightarrow$ $x(\theta) \in S$ as $r \rightarrow 1$, we conclude that there exists $\widehat{x}(\theta) \in \widehat{S}$ such that $\widehat{\lambda}_{\theta}(r) \rightarrow \widehat{x}(\theta)$. But any point of $\widehat{S}$ is an image of an interior point of $D$ under the uniformizing map $\widehat{\Phi}$, and not a boundary value of this map, a contradiction.

\subsection{Boundary values of the complex length.}

Let $\pi_{V}: L C_{\mathcal{W}} \rightarrow V, \pi_{U}: L C_{\mathcal{W}} \rightarrow U$, be the same as before.

Lemma 3. The map $\pi_{V}: L C_{\mathcal{W}} \rightarrow V$ is tame on discs.

Proof: Consider a holomorphic nonconstant map $\Phi: D \rightarrow L C_{\mathcal{W}}$, and define the pull-back of the complex length $L$ to $D$. Note that the length $L$ is well defined on the universal cover $\widehat{L C}_{\mathcal{W}}$ rather than on $L C_{\mathcal{W}}$, see 
Subsection 3.3. Let $\widehat{\Phi}$ be any lift of $\Phi$ to a map $D \rightarrow \widehat{L C}_{\mathcal{W}}: \widehat{\pi} \circ \widehat{\Phi}=\Phi$. Define

$$
\widetilde{L}=L \circ \widehat{\Phi}
$$

Lemma 4. Consider $\widetilde{L}$, the complex length vector function lifted to the unit disc $D$, see (18). There is an open dense set $\Omega$ on the boundary $S^{1}=$ $\partial D$ through which the function $\widetilde{L}$ may be analytically extended. The complement $\Lambda=S^{1} \backslash \Omega$ is closed and has measure zero.

Proof: Consider $n+1$ holomorphic functions in $D$ : the function

$$
\widetilde{\alpha}=\pi_{V} \circ \Phi
$$

and the functions $\widetilde{L}_{1}, \ldots, \widetilde{L}_{n}$, the components of $\widetilde{L}$. These components range in a halfplane $\Re \zeta \leq C$ for some $C$. Hence, for appropriate $c$, the functions $l_{j}=1 /\left(\widetilde{L}_{j}+c\right)$ are all holomorphic and bounded, as well as $\widetilde{\alpha}$ is. By the Fatou Theorem, they all have radial limits at almost every point of the boundary circle $S^{1}$. Denote by $\Lambda_{\infty}$ the set of those $\theta$ for which there exists $j$ such that either $l_{j}\left(r e^{i \theta}\right) \rightarrow 0$ or $l_{j}\left(r e^{i \theta}\right)$ has no radial limit at all as $r \rightarrow 1$. By the Fatou and Privalov Theorems, mes $\Lambda_{\infty}=0$. Note that if $\theta \notin \Lambda_{\infty}$, all the functions $\widetilde{L}_{j}$ have a finite radial limit at $e^{i \theta}$.

Denote by $P$ (of polycycle) the set of those $\alpha \in V$ for which $\mathcal{F}_{\alpha}$ has a polycycle close to $\gamma$. Recall that the set of these points is at most countable. Denote by $\Lambda_{P}$ the set $\left\{\theta \mid \lim _{r \rightarrow 1} \widetilde{\alpha}\left(r e^{i \theta}\right) \in P\right\}$. Again by the Privalov Theorem

$$
\operatorname{mes} \Lambda_{P}=0 \text {. }
$$

Let us now prove that the vector function $\widetilde{L}$ may be extended to any point of the set $S^{1} \backslash \Lambda_{P} \backslash \Lambda_{\infty}$. This will imply that this set is open. The previous arguments show that it is of measure zero.

Let $\theta \in S^{1} \backslash \Lambda_{P} \backslash \Lambda_{\infty}$.

Then all the functions $\widetilde{L}_{j}$ have a radial limit along $\left\{r e^{i \theta}\right\}$. By Theorem 2 , the length of the cycles $\gamma_{\alpha(r)}$, where $\alpha(r)=\pi_{V} \Phi\left(r e^{i \theta}\right)$, stays bounded as $r \rightarrow 1$. By Lemma 1, the family of complex limit cycles $\gamma_{\alpha(r)}$ tends to a complex cycle or polycycle $\gamma^{0}$. But the latter case is excluded because $e^{i \theta} \notin \Lambda_{P}$. Hence, $\gamma^{0}$ is a complex cycle, and the function $\widetilde{L}$ may be extended through the point $e^{i \theta}$. This proves Lemma 4.

Lemma 4 implies Lemma 3.

Together, Lemma 2 and Lemma 3 imply Theorem 1. 


\title{
References
}

[1] G. T. Buzzard, S. L. Hruska, and Yu. Ilyashenko, KupkaSmale theorem for polynomial automorphisms of $\mathbb{C}^{2}$ and persistence of heteroclinic intersections, Invent. Math. 161(1) (2005), 45-89. DOI : $10.1007 / \mathrm{s} 00222-004-0418-8$.

[2] G. Calsamiglia, B. Deroin, S. Frankel, and A. Guillot, Singular sets of holonomy maps for algebraic foliations, J. Eur. Math. Soc. (JEMS) 15(3) (2013), 1067-1099. DOI: 10.4171/JEMS/ 386.

[3] YU. S. IL'YASHENKo, Limit cycles of polynomial vector fields with nondegenerate singular points on the real plane, (Russian), Funktsional. Anal. i Prilozhen. 18(3) (1984), 32-42; translation in: Functional Anal. Appl. 18(3) (1984), 199-209.

[4] Yu. S. IL'Yashenko, Persistence theorems and simultaneous uniformization, (Russian), Tr. Mat. Inst. Steklova 254 (2006), Nelinein. Anal. Differ. Uravn., 196-214; translation in: Proc. Steklov Inst. Math. 3(254) (2006), 184-200.

[5] YU. IL'YASHENKO, Some open problems in real and complex dynamical systems, Nonlinearity 21(7) (2008), T101-T107. DOI : 10.1088/ 0951-7715/21/7/T01.

[6] Yu. Il'yashenko and S. Yakovenko, Finite cyclicity of elementary polycycles in generic families, in: "Concerning the Hilbert 16th problem", Amer. Math. Soc. Transl. Ser. 2 165, Amer. Math. Soc., Providence, RI, 1995, pp. 21-95.

[7] I. G. Petrovskil and E. M. Landis, On the number of limit cycles of the equation $d y / d x=P(x, y) / Q(x, y)$, where $P$ and $Q$ are polynomials of 2nd degree, (Russian), Mat. Sb. N.S. 37(79) (1955), 209-250.

\author{
Cornell University \\ Ithaca, NY 14853 \\ USA \\ and \\ Moscow State and Independent Universities \\ Steklov Mathematical Institute \\ National Research University Higher School of Economics \\ 119002 Moscow \\ Russia \\ E-mail address: yulijs@gmail.com
}

